\title{
The abundances of $\mathrm{A} / \mathrm{F}$ and $\mathrm{Am} / \mathrm{Fm}$ stars in open clusters
}

\author{
Richard Monier and Olivier Richard \\ GRAAL, Universite Montpellier II, Place E. Bataillon, 34095 Montpellier, France \\ email: Richard.Monier@graal.univ-montp2.fr, Olivier.Richard@graal.univ-montp2.fr
}

\begin{abstract}
A short review on abundance determinations of A and F dwarfs in the Hyades is presented. The spectroscopy was carried out with AURELIE, a monorder spectrograph at the Observatoire de Haute Provence (France) at a resolving power close to 40000 with Signal-toNoise ratios varying from 100 to 300 . Abundances of 11 chemical elements have been derived by using Takeda's (1995) procedure.
\end{abstract}

Keywords. Stars: abundances, stars: chemically peculiar, Galaxy:open clusters and associations: individual: Hyades, diffusion

\section{Abundance determinations of $A$ and $F$ stars in the Hyades}

Very few studies have so far addressed the chemical composition of A stars in the Hyades. They usually have focused on a very limited numbers of stars not necessarily well distributed in mass along the main sequence. For the Hyades cluster, Burkhart \& Coupry (1989) have derived the abundances of Li, Al, Si and Fe for 5 Am and 1 A stars. Takeda \& Sadakane (1997) found the abundances of $\mathrm{O}$ and Fe for $8 \mathrm{Am}$ and 10 normal stars and Hui-Bon-Hoa \& Alecian (1998) those of Mg, Ca, Sc, Cr, Fe and Ni for 4 Am and 2 normal A dwarfs. Varenne \& Monier (1999) have determined the abundances of 11 chemical elements (C I, O I, Na I, Mg I, Si I, Ca I, Sc II, Fe I, Ni I, Y II, Ba II) for a much larger sample of stars: $19 \mathrm{~A} / \mathrm{Am}$ dwarfs and $29 \mathrm{~F}$ dwarfs using AURELIE spectra centered at three wavelengths $(6160 \AA, 5080 \AA$ and $5530 \AA)$. These stars are regularly distributed in spectral type along the main sequence to sample the expected masses in a uniform manner. All these stars were analysed in a uniform manner using spectrum synthesis as a few of them are fast rotators. For the Hyades, Varenne \& Monier (1999) found large star-to-star variations for the normal A stars in particular for $\mathrm{O}, \mathrm{Na}, \mathrm{Ni}, \mathrm{Y}$ and $\mathrm{Ba}$. The Am stars are almost all deficient in $\mathrm{Sc}$ and $\mathrm{Ca}$ and overabundant in $\mathrm{Fe}, \mathrm{Ni}$, $\mathrm{Y}$ and $\mathrm{Ba}$ and also show star-to-star variations. In contrast, the $\mathrm{F}$ stars show very little scatter in their abundances. The A stars show a much larger scatter than the $\mathrm{F}$ stars on graphs displaying the abundances of individual elements $[\mathrm{X} / \mathrm{H}]$ versus the effective temperature $\left(T_{\text {eff }}\right)$. No convincing anticorrelation between the abundances and rotational velocity $\left(v_{e} \sin i\right)$ was found.

\section{Conclusions}

In the Hyades, we find evidence for large star-to-star variations in the normal A stars and in the Am stars as in Coma Berenices. There is very little scatter among the F dwarfs in both clusters. We believe that the differences in abundances among the A stars born from the same original interstellar matter may be the signature of the occurence of transport processes in their interiors.

We are currently observing A/Am and F/Fm stars in other open clusters of various 
Table 1. Abundances relative to hydrogen $\left[\frac{X}{H}\right]$ for the A stars in the Hyades. Values in brackets are uncertain.

\begin{tabular}{ccccccccccccc}
\hline VB & SpT & C I & O I & Na I & Mg I & Si I & Ca I & Sc II & Fe I & Ni I & Y II & Ba II \\
\hline 38 & A3m & & & & -0.22 & & -0.60 & -1.31 & 0.12 & 0.10 & & \\
47 & A7V/A8V & 0.07 & -0.11 & 0.26 & -0.13 & 0.07 & 0.01 & 0.11 & -0.06 & 0.20 & 0.30 & 0.02 \\
54 & A7IV/A6V & 0.05 & 0.08 & 0.60 & 0.04 & 0.13 & 0.07 & 0.13 & 0.07 & 0.17 & -0.10 & -0.05 \\
55 & A7V/A6V & & 0.10 & 0.19 & -0.64 & -0.41 & -0.36 & 0.06 & -0.30 & -0.52 & & -0.71 \\
56 & A2IV/Am & -0.20 & -0.34 & 0.50 & 0.08 & 0.13 & -0.02 & -0.99 & 0.28 & 0.80 & 0.76 & 0.99 \\
60 & A8V & & 0.07 & -0.28 & -0.79 & -0.60 & -0.46 & 0.41 & -0.57 & -0.54 & & -0.94 \\
67 & A3m & -0.55 & -0.46 & 0.19 & -0.16 & 0.03 & -0.47 & $\leqslant-1.2$ & 0.19 & 0.33 & 0.70 & 0.77 \\
72 & A7III/A7IV & -0.15 & -0.19 & -0.40 & -0.16 & 0.11 & -0.14 & -0.05 & -0.13 & 0.12 & 0.00 & -0.20 \\
74 & A7V/A5m & -0.10 & -0.29 & -0.06 & -0.28 & 0.22 & -0.31 & $\leqslant-1.5$ & 0.17 & 0.49 & 0.50 & 0.78 \\
82 & A6IV/A7V & & -0.37 & 0.08 & -0.14 & 0.05 & -0.03 & 0.11 & 0.07 & 0.48 & 0.74 & 0.48 \\
83 & A5m & -0.20 & -0.43 & 0.23 & -0.01 & 0.06 & -0.26 & -0.47 & 0.18 & 0.63 & 0.80 & 1.21 \\
95 & A8V/A7V & $(-0.70)$ & -0.18 & 0.54 & -0.01 & -0.05 & -0.08 & 0.22 & 0.10 & 0.27 & & 0.70 \\
104 & A6V & 0.00 & 0.09 & 0.54 & 0.05 & 0.05 & 0.16 & 0.17 & 0.02 & 0.14 & 0.11 & -0.03 \\
107 & A5m/A9III & & -0.08 & 0.45 & 0.14 & 0.22 & 0.15 & 0.27 & 0.23 & 0.52 & 0.60 & 0.57 \\
108 & A5V/A6V & & 0.02 & & -0.15 & -0.11 & 0.12 & 0.21 & -0.05 & -0.05 & & -0.07 \\
112 & A2m & $(-0.75)$ & -0.81 & 0.41 & -0.03 & 0.17 & -0.56 & $\leqslant-1.5$ & 0.38 & 0.78 & 1.17 & 1.48 \\
123 & A7V/A9V & & & & -0.02 & & -0.25 & 0.10 & -0.24 & & & \\
129 & A7V & $(0.00)$ & 0.03 & 0.36 & 0.05 & 0.12 & 0.02 & 0.14 & 0.00 & 0.08 & & -0.03 \\
131 & A5m & -0.44 & -0.49 & 0.16 & -0.20 & 0.09 & -0.23 & -0.61 & 0.19 & 0.64 & 0.89 & 1.19 \\
& & & & & & & & & & & & \\
$\alpha \mathrm{CMi}$ & F5IV/V & 0.12 & -0.12 & 0.05 & 0.06 & 0.03 & -0.05 & 0.04 & -0.02 & -0.01 & -0.07 & 0.03 \\
\hline
\end{tabular}

ages with 2-meter class telescopes (Monier \& Richard 2005) and will soon start observing more distant clusters with UVES.

\section{References}

Burkhart C., Coupry M.F., 1989, A\&A 220, 197

Hui-Bon-Hoa A., Alecian G., 1998, A\& $A$ 332, 224

Monier R., Richard O., 2005 These Proceedings, 209

Takeda Y., Sadakane K., 1997, PASJ 49, 367

Varenne O., Monier R., 1999, A\&A 351, 247 